\title{
TUBELESS PERCUTANEOUS NEPHROLITHOTOMY;
}

\section{ANALGESIA REQUIREMENT IN CONVENTIONAL AND TUBELESS PERCUTANEOUS NEPHROLITHOTOMY: A COMPARISON}

Dr. Muhammad Nasir Ibrahim, Dr. Muhammad Muzammil Tahir, Dr. Amjad Ali Saddique, Dr. Mohammad Usman Khan

ABSTRACT... Revolutionary advances in the minimally invasive and non invasive management of stone disease over the past two decades have greatly facilitated the removal of stones. Renal stone management moved from open surgery to minimally invasive procedures with the aim of achieving maximum stone clearance with the least morbidity and mortality. Percutaneous nephrolithotomy (PCNL) remains the most efficient procedure in all patient groups. Tubeless PCNL is increasing in popularity and the technique of percutaneous nephrolithotomy is in constant evolution. Nephrostomy tube has been implicated in causing postoperative discomfort or pain and morbidity. Nephrostomy-free or tubeless PCNL reduces postoperative pain and analgesia related to the drainage tube. Objective: To compare mean analgesia requirement with tubeless PCNL and standard PCNL in renal stone patients Study design: Randomized controlled trial conducted at Shaikh Zayed Hospital Lahore from July 2010 to January 2011. Methods: 100 patients were divided into two groups randomly by using random number tables i.e. 50 patients in group A and 50 patients in group B. Group A patients were operated by standard PCNL method i.e. with Placement of nephrostomy tube and ureteric catheter and group B were operated by tubeless PCNL i.e. without nephrostomy tube but with ureteric catheter. The data was collected on attached predesigned computer based proforma. Results: The analgesic dose requirement in Group A was $116.50 \pm 26.55 \mathrm{mg}$ and analgesic dose requirement in Group B was $73.00 \pm 30.24 \mathrm{mg}$ of pethidine. So, analgesia requirement in both groups showed a significant difference $(p<0.05)$. Conclusions: Tubeless PCNL can be used with a favorable outcome in renal stone patients, with the potential advantage of decreased analgesia requirement.

Key words: Nephrostomy tube, PCNL, Tubeless PCNL.

Article Citation

Ibrahim MN, Tahir MM, Saddique AA, Khan MU. Tubeless Percutaneous Nephrolithotomy; Analgesia requirement in conventional and tubeless percutaneous nephrolithotomy: A comparison. Professional Med J 20(5): 787-792.

\section{INTRODUCTION}

Although Stone disease is one of the most common afflictions of modern society, it has been described since antiquity. Poverty, high population density, poor nutritional status, inadequate health facilities, hot climate are few factors promoting stone disease. In this region $60-65 \%$ of the patients form calcium oxalate stones, $15-30 \%$ uric acid and $10-15 \%$ struvite stones. Ammonium hydrogen urate is found in $30 \%$ of renal calculi in children and $3 \%$ in adults ${ }^{1}$.

Management of urolithiasis constitutes $40-50 \%$ of the urological workload in a hospital. Initially open surgery was the only treatment for renal calculi. Since the clinical introduction of extracorporeal shock wave lithotripsy (ESWL) and endourological techniques, the management of urolithiasis changed completely. The high risk of stone recurrence also favors minimally invasive treatment rather than repeated open surgery. It was in 1976 when Dr. Fernstrom and Dr. Johanson first described the procedure of $\mathrm{PCNL}^{2}$. Certain conditions have specific indications for PCNL as in cystine stones, lower pole calyceal stone with narrow, long infundibulum, acute infundibulo-pelvic angle and some upper ureteric stones ${ }^{3}$. Moreover, American Urology Association (AUA) Nephrolithiasis Clinical Guideline Panel has recommended PCNL as first line of treatment for staghorn calculi followed by ESWL or repeat PCNL as needed ${ }^{4}$.

The procedure has been practiced for treating urolithiasis in adults for about a decade in Pakistan. In a local series, stone clearance or planned debulking by PCNL has been reported up to $97 \%$, this compares equally to other international studies. PCNL is a valuable treatment option even for complete staghorn stones with a stone-free rate approaching that of open surgery ${ }^{5}$.

Tubeless PCNL is increasing in popularity and the technique of percutaneous nephrolithotomy is in constant evolution. Percutaneous nephrolithotomy 
with conventional post operative nephrostomy tube drainage of the kidney is a routine approach in patients with renal calculi. Nephrostomy tube has been implicated in causing postoperative discomfort and morbidity, prolonged hospital stay and continuous urinary leakage ${ }^{6}$. Tubeless PCNL in selected patients is a secure, effective procedure, causes less morbidity and does not compromise patient safety.

Nephrostomy-free or tubeless PCNL reduces postoperative urinary leakage, analgesia requirement and local pain related to the drainage tube. It also minimizes hospital stay ${ }^{7}$. PCNL is not free from complications, fever, blood loss, puncture site wound, infection, ureteric obstruction due to stone fragments, hypertrophied puncture site scar, and chest complications have been reported ${ }^{8}$.

Analgesia requirement will be measured as total dose of Pethidine in $\mathrm{mg} / 24$ hours to maintain Visual analogue scale (VAS) score $<20$, with scale, 0-100. Based on hypothesis, that mean analgesia requirement will be less with tubeless PCNL than standard PCNL.

\section{MATERIALS AND METHODS}

This non probability purposive sampling, randomized controlled study was conducted in the department of Urology, Sheikh Zayed Hospital, Lahore, in six months duration from July 2010 to January 2011, with sample size of 100 cases. Our Inclusion criteria was, Patients of both sexes, more than 15 Years of age, Patients with renal stone larger than $2 \mathrm{~cm}$ in size (size will be measured by ultrasound), Stone larger than $1.5 \mathrm{~cm}$ in lower pole calyx. (size and location as seen on IVU), and patients who required single or two puncture sites. Exclusion criteria was, patients with bleeding disorders (INR>1.5), Pregnant female, residual stones, and $>2$ puncture sites.

100 patients, fulfilling the inclusion \& exclusion criteria and after taking informed consent, obtaining demographic information were divided into two groups randomly by using random number tables i.e. 50 patients in group $A$ and 50 patients in group $B$. Group A patients were operated by standard PCNL method i.e. placement of nephrostomy tube and ureteric catheter and group B were operated by tubeless PCNL i.e. without nephrostomy tube but with ureteric catheter.

Patients were given pre operative medication prior to shifting to operating suite to allay anxiety. General anesthesia was given. Retrograde pyelography was done routinely in all patients to delineate the anatomy and to fill the pelivalceal system with contrast so as to facilitate the percutaneous access. An open ureteric catheter was left in the ureter with its tip preferably just above the pelviureteric junction. also if required to refill contrast at the time of percutaneous access. Patient was changed to prone. Operating site was prepared with povidone ${ }^{\circledR}$ paint as disinfectant and draped. Access was acquired through upper, middle or lower caliceal systems in accordance with stone burden, location and dilatation of particular calyx.

Puncture for PCNL tract was made with 16G spinal needle with Kellet needle 19G. Then a guide wire 0.032 inch diameter was threaded in through the spinal needle into the pelvicalyceal system. The tract was then gradually dilated first with vascular dilators followed by metallic dilator and graduated metallic dilators one on other. While holding the olive tip metallic dilator firm and guide wire in the line and under traction. After the last metallic dilator, Amplatz sheath was passed over the whole set of dilators down to cover the olive tip. Amplatz sheath secures the nephroscope in the PCNL tract. As soon as the position of the amplatz is confirmed, metallic dilator set was removed while retaining the guide wire in Nephroscope with sheath $26 \mathrm{Fr}$ passed through $30 \mathrm{Fr}$ Bard $\circledast$ amplatz sheath. Guide wire was retained till the end. Stones were targeted and fragmented with pneumatic lithoclast interchangeably as single or 
multiple fire. At the end of procedure a search for any residual stone was made through image intensifier. Finally a PCN drainage tube was left through the amplatz. At our centre $16 \mathrm{Fr}$ foley catheter without balloon dilatation, is used as nephrostomy tube. Nephrostomy tube was placed in Group A patients only.

After the completion of procedure nephrostomy tube and ureteric catheter were removed after 24 hours and 48 hours respectively. Patients from both groups were counseled about pain and demand of analgesia according to visual analogue scale(VAS). Injection Pethidine was injected to keep the VAS $<20$ and total dose of pethidine was calculated in both groups after 24 hours. All Patients underwent X-Ray KUB to see residual stones, before discharge. Both groups were compared post operatively in terms of analgesia requirement. The collected information was transferred to SPSS version 12 analyzed. Analgesic dose in both groups was reported by using mean \pm S.D and comparison was made by using $T$ test. $P$ value $\leq 0.05$ was considered as significant.

\section{RESULTS}

\section{Age and Sex}

There were 50 patients in Group A and 50 patients in Group B. The mean age of patients in group A was 34.18 and in Group B 32.2. There were 32 male patients and 18 female patients in Group A. While in Group B male patients were 27 and female patients were 23 as shown in table no. I

\begin{tabular}{|l|c|c|}
\hline & Group A $(\mathbf{n = 5 0 )}$ & Group B $(\mathbf{n = 5 0 )}$ \\
\hline $\begin{array}{l}\text { Age (years) } \\
\text { Mean SD }\end{array}$ & $34.18 \pm 9.6$ & $32.2 \pm 12.0$ \\
\hline Gender (M:F) & $\begin{array}{c}32: 18 \\
(64: 36 \%)\end{array}$ & $\begin{array}{c}27: 23 \\
(54: 46 \%)\end{array}$ \\
\multicolumn{3}{|c|}{$\begin{array}{c}\text { Table-I. } \\
\text { Demographic profile }\end{array}$} \\
\multicolumn{2}{|c|}{ Group A: standard PCNL Group B: tubeless PCNL }
\end{tabular}

\section{DISCUSSION}

In the era of minimally invasive and non invasive techniques for treating renal stones although ESWL has revolutionized the management of urinary stones, PCNL still plays an important role in the treatment of large or multiple kidney stones. In recent decades, endoscopic technology and operative techniques have consistently advanced, which has increased the success rate $(>90 \%)$ of PCNL and decreased the associated complications and morbidity ${ }^{10}$.

Previously, it was thought that one of the advantages of placing nephrostomy tube is tract homeostasis, but, if the bleeding is not too much, leaving nephrostomy tube in place after PCNL in this regard does not seem to be beneficial; and its omission does not put patients at any additional risk"11.

However, based on the concept that the purpose of the tube is only to maintain adequate drainage of the kidney, a tubeless approach has been developed by placing a ureteral stent or catheter to provide drainage

\begin{tabular}{|c|c|c|c|c|c|c|c|}
\hline & Group & N & Mean & $\begin{array}{c}\text { Std. } \\
\text { deviation }\end{array}$ & $\begin{array}{c}\text { Std. err or } \\
\text { mean }\end{array}$ & $P$ value & Significance \\
\hline \multirow{2}{*}{$\begin{array}{l}\text { Analgesia } \\
\quad(\mathrm{mg})\end{array}$} & A & 50 & 16.50 & 26.55 & 3.75 & \multirow{2}{*}{0.000} & \multirow{2}{*}{ Significant } \\
\hline & B & 50 & 73.00 & 30.24 & 4.28 & & \\
\hline \multicolumn{8}{|c|}{$\begin{array}{l}\text { Table-II. Results and P value } \\
\text { df: } 98 \quad 96.379 \quad \text { Confidence interval: } 95 \% \\
\text { andard PCNL } \\
\text { Group B; tubeless PCNL }\end{array}$} \\
\hline
\end{tabular}


after PCNL in lieu of a nephrostomy tube. However, in recent years, the procedure has been modified to what has been called, tubeless PCNL, in which nephrostomy tube is replaced with internal drainage provided by a double-J stent or a ureteral catheter. It may be interesting to note that the idea of tubeless existed even in the early years of evolution of PCNL. In 1984, Wickham published the results of 100 patients in which no internal or external drainage tubes were used at the conclusion of case. Authors stated that with this approach, patients could leave the hospital within 24 hour and the procedure was safe and efficient with a shorter hospital stay ${ }^{13}$.

However, subsequently Winfield et al reported two patients with complications of premature nephrostomy-tube removal after the extraction of simple upper-tract calculi, who experienced serious hemorrhage and marked urinary extravasation necessitating transfusion, internal stenting, and prolonged hospitalization. They recommended that nephrostomy tube drainage should be provided during the first 24 to 48 hour after percutaneous stone extraction which subsequently became the standard practice for PCNL worldwide ${ }^{12}$.

In 1997, Bellman and associates challenged the conventional PCNL. Their tubeless procedure involved the placement of an internal ureteral stent without any nephrostomy tubes. The study group consisted of 50 patients, who were compared with a control group of 50 patients undergoing percutaneous renal surgery with the standard nephrostomy tube. The hospitalization time, analgesia requirements, time to return to normal activities, and cost were significantly less with this new technique ${ }^{14}$. A randomized comparison of tubeless and standared PCNL by Agrawal showed the mean analgesia requirement for standard PCNL (meperidine $126.5+/-33.3 \mathrm{mg}$ ) was significantly more compared with tubeless group (meperidine $81.7+/-24.5 \mathrm{mg})(\mathrm{P}<0.01)^{9}$. This study was conducted to compare mean analgesia requirement with tubeless PCNL and standard PCNL in renal stone patients. Shah NH, in his 454 patient study gives less post operative pain and analgesia requirement $(p<0.001)^{16}$. In another study carried out in Pakistan by Husain I, give analgesia requirement 50 , 47.3 and $21.13 \mathrm{mg}$ for large bore, small bore nephrostomy tube and tubeless PCNL respectively ${ }^{18}$.

In the largest prospective randomized trial published , in 202 patients, tubeless PCNL (101 patients) was found to have significant advantages over standard PCNL (101 patients) in terms of postoperative pain, analgesia requirement, morbidity, hospital stay, and period of convalescence. The average visual analogue scale (VAS) pain score on postoperative day 1 for tubeless group patients was $31 \mathrm{~mm}$ compared with 59 $\mathrm{mm}$ in standard PCNL $(P<0.01)^{15}$. Tubeless PCNL was safe and effective even in patients with a solitary kidney, or with three renal access tracts or supracostal access, or with deranged renal values and in those requiring bilateral simultaneous PCNL. The literature review suggested a need for prospective, randomized studies to evaluate the role of fibrin sealant and/or cauterization of the nephrostomy tract in tubeless PCNL $^{16}$.

Our study also shows that PCNL in "tubeless" fashion does'nt put patients at risk. Rather it is less morbid and safe as it requires less analgesia (pethidine) $73 \mathrm{mg}$, compare to $116.5 \mathrm{mg} / \mathrm{dl}$. So less incidence of narcotics induced side effects are observed. The results of my study are comparable to the result of other studies especially in the context of analgesia requirement. That's why nephrostomy-free or tubeless PCNL minimizes hospital stay, the majority of patients were discharged from the hospital in fewer than 24 hours.

Advances/modification in surgery has been a part of history. So in future there will be more trend towards totally tubeless PCNL i.e. without nephrostomy tube and internal drainage (stents). 
Several centers have reported their experience with bilateral tubeless PCNL. Shah and colleagues found no increase in the complication rate when comparing their series of 10 bilateral tubeless PCNLs with 10 prior procedures with nephrostomy tube ${ }^{17}$.

Several retrospective studies have shown that application of tube less can be extended even in patients with multiple, complex and staghorn stones, concurrent UPJ obstruction, solitary kidney, previous ipsilateral open surgery, raised serum creatinine level, with multiple or supracostal tracts, and in patients undergoing bilateral synchronous PCNL. The technique has been successful in obese patients, children, and patients with recurrent stones after open surgery. However, for all these extended indications, the available evidence is insufficient, and needs to be substantiated by prospective randomized trials ${ }^{15}$.

\section{CONCLUSIONS}

Tubeless PCNL can be used with a favorable outcome in renal stone patients with the potential advantages of decreased analgesia requirement and less patient morbidity.

\section{Copyright@ 15 Sep, 2013.}

\section{REFERENCES}

1. Rizvi S A H, Naqvi SAA, Hussain Z, Mehdi H, Khalid R. The management of stone disease. BJU 2002; 89:1 62.

2. Fernstrom I, Johannson B. Percutaneous Pyelolithoitomy: A new extraction tehnique. Scand J Urol Nephrol 1976;10:257-9.

3. Monish Aron, Rajiv Goel, Pawan.K.Keser, Narmada Gupta, Upper Pole access for complex lower pole renal calculi. BJU Int 2004; 94(6):849-51.

4. Cranidis A I, Karayannis A A, Delakas D S, Livadas C E. Cystine stones: the efficacy of percutaneous and shock wave lithotripsy. Urol Int $1996 ; 56: 180-3$.

5. Al-Kohlany KM, Shokeir AA, Mosbah A, etal. Treatment of complete staghorn stones. A prospective randomized comparison of open surgery versus percutaneous nephrolithotomy. J Urol 2005;17:3469.

6. Basiri A, Ahmadnia H, Moghaddam SM. The efficacy of conventional PCNL and two modifications to standard procedure. J Pak Med Assoc 2006; 56:3025 .

7. Singh I, Singh A, Mittal G. Tubeless percutaneous nephrolithotomy: is it really less morbid? J Endourol 2008; 22:427-34.

8. Tahir M, Khan G, Khan U; Is tubeless PCNL is really worthwhile; PPMJ; 2008; 18(4): 133-5.

9. Agrawal MS, Agrawal M, Gupta A, Bansal S, Yadav A Goyal J. A randomized comparison of tubeless and standard percutaneous nephrolithotomy. J Endourol 2008; 22:439-42.

10. Marguet CG, Springhart WP, Tan YH, Patel A, Undre S, Albala DM, et al. Simultaneous combined use of flexible ureteroscopy and percutaneous nephrolithotomy to reduce the number of access tracts in the management of complex renal calculi. BJU Int 2005;96:1097-100.

11. Etemadian M, Soleimani MJ, Haghighi R, Zeighami MR, Najimi N. Does Bleeding During Percutaneous Nephrolithotomy Necessitate keeping the Nephrostomy Tube? A Randomized Controlled Clinical Trial. Urol J 2011;8:21-6.

12. Winfield HN, Weyman P, Clayman RV. Percutaneous nephrostolithotomy: Complications of premature nephrostomy tube removal. J Urol. 1986;136:77-9.

13. Wickham JE, Miller RA, Kellett MJ, Payne SR Percutaneous nephrolithotomy: One stage or two? Br JUrol. 1984;56:582-5

14. Bellman GC, Davidoff R, Candela J, Gerspach J, Kurtz S, Stout $L$. Tubeless percutaneous renal surgery. J Urol 1997;157:1578-82.

15. Agrawal MS, Agrawal M. Tubeless percutaneous nephrolithotomy. Indian J Urol 2010 Jan-Mar; 26(1):16-24.

16. Shah HN, Kausik VB, Hegde SS, Shah JN, Bansal MB 
Tubeless percutaneous nephrolithotomy: a prospective feasibility study and review of previous reports. BJU Int 2005 Oct;96(6):879-83

17. Weld KJ, Wake RW. Simultaneous bilateral tubeless percutaneous nephrolithotomy. Urology. 2000;56:1057.
18. Husain I, Tahir MM, Ashraf S, Khan MU; Percutaneous Nephrolithatomy; Randomised comparison of large bore, small bore and tubeless; Professional Med J; Oct-Dec 2011; 18(4); 575-580.

\section{AUTHOR(S):}

1. DR. MUHAMMAD NASIR IBRAHIM

Registrar Urology Department

Shaikh Zayed Hospital Lahore.

2. DR. MUHAMMAD MUZAMMIL TAHIR

Associate Professor Urology

FPGMI/Shaikh Zayed Hospital Lahore

3. DR. AMJAD ALI SADDIQUE

Consultant Urologist

Govt Social Security Hospital Lahore.

4. Dr. Mohammad Usman Khan

Professor of Urology

FPGMI/Shaikh Zayed Hospital Lahore
Correspondence Address:

Dr. Muhammad Nasir Ibrahim

Registrar Urology Department

Shaikh Zayed Hospital Lahore.

dr_muzamil@yahoo.com

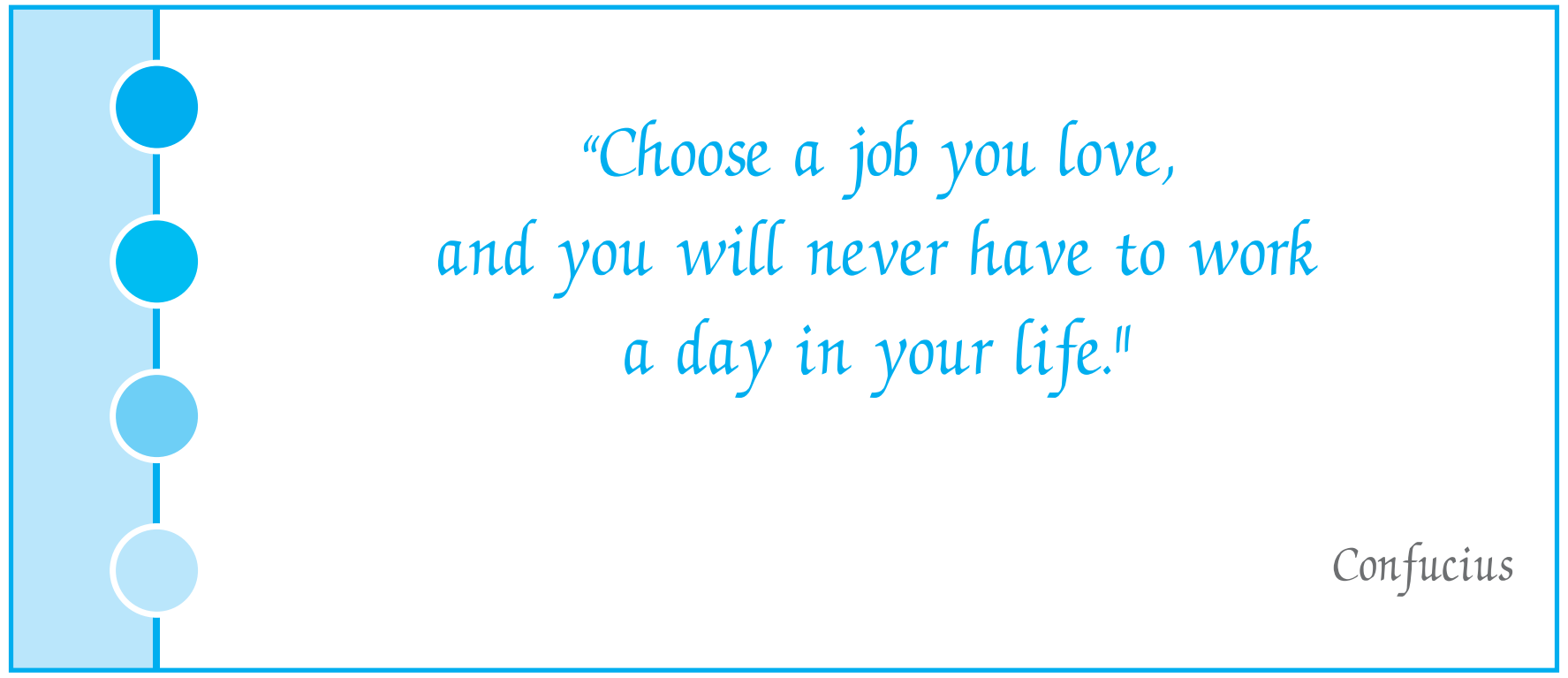

